\title{
Modal analysis of a stator of Double Rotor Traveling Wave Rotary Ultrasonic Motor (DTRUM) (Single Degree of Freedom)
}

\author{
Abdulrahaman Shuaibu Ahmad ${ }^{1}$, Prof. Dr. Qing $\mathrm{He}^{2}$, \\ Abdulrrahman Ahmad Umar ${ }^{3}$, Saidu Bello Abubakar ${ }^{4}$, \\ ${ }^{I}$ (Department of mechanical engineering and automation, Liaoning University of technology, China) \\ ${ }^{2}$ (Department of mechanical engineering and automation, Liaoning University of technology, China) \\ ${ }^{3}$ (Department of mechanical engineering and automation, Liaoning University of technology, China) \\ ${ }^{4}$ (Department of mechanical engineering, University of Malaysia (UTM), Malaysia)
}

\begin{abstract}
This paper outlined the modal analysis of a Double Rotor Bar-type Rotary Traveling Wave Ultrasonic Motor (DBTRUM) with single degree of freedom (SDOF). The first bending vibration mode generated from the inverse piezoelectric effect produces traveling wave in the stator, the traveling wave generated in the stator is converted to rotary motion on the two rotors by the use of friction layer between the rotor and the stator surfaces. The system was first designed theoretically after which it was then simulated with finite element method software (ANSYS) to obtain the modal frequency and mode shape.
\end{abstract}

Keywords: Rotor, Stator, Ultrasonic Motor, Traveling wave, Frequency, Bending mode, mode shape.

\section{Introduction}

The double rotor bar-type traveling wave rotary ultrasonic motor (DBTRUM) is an important actuator which promise a vast potential market in micro-robot, precision instruments, medical equipment and other industrial areas [1], [2]. There are certain requirements for the design of the stator of DBTRUM. First of all, the stator must have a proper operating modal frequency, [3]. A too low modal frequency will lead to the noise, lower vibration velocity and limit output power of the motor. If the modal frequency is too high, energy loss will increase inside the motor. So the efficiency of the motor will reduce. Experiments show that the proper modal frequency of the motor should be in the range from $30 \mathrm{kHz}$ to $10 \mathrm{kHz}$. Secondly, there is a larger difference between the operating mode frequency and other mode frequencies for avoiding the modal interferences. There are also some requirements for the formation of the required mode shape when designing DBTRUM [4], [5]. the first bending vibration mode is adopted for this system, it is formed by exciting a sinusoidal alternating voltage to one part of the piezoelectric ceramic ring at the center of the stator with sin $\omega t$ and the other part with $\cos \omega t$ (The excitation frequency is close to the resonance frequency of the first bending modes) [6], the stator will vibrate in the left and right, front and behind directions respectively, these two perpendicular bending modes will be excited when the excitation voltages are applied to the two piezoelectric ceramic sets, and these modes are composed to a rotating bending mode (one traveling wave). The stator being a key part of DBTRUM, whose dynamic characteristics have a very important effect on the performance of DBTRUM. So the modal frequency and modal shape of an ultrasonic motor must be primarily designed, and the polarization pattern of piezoelectric ceramics should be given proper attention.

Usually the bending Bmn is applied for traveling wave ultrasonic motors, where $\mathrm{m}$ and $\mathrm{n}$ indicates the number of nodal circle and nodal diameters, respectively [7], [8]. It is important to design operating modes (two standing waves), which forms traveling wave in a stator. The bigger $\mathrm{n}$ depicts a higher order mode, which leads to smaller vibration amplitude of stator at the same excitation. On the contrary, the smaller $\mathrm{n}$ indicates a lower order mode. Note worthily noise in TRUM will be produced if the modal frequency is below $20 \mathrm{kHz}$. Therefore the operating frequency of TRUM lies in the range from $30 \mathrm{kHz}$ to $50 \mathrm{kHz}$ for the TRUM stator with smaller radius than $40 \mathrm{~mm}$.

A hollow bar of with inner diameter $0.011 \mathrm{~m}$, outer diameter $0.030 \mathrm{~m}$, and length $0.039 \mathrm{~m}$ was used, two sets of piezoelectric ceramic rings were used to design the prototype of this system. The first bending mode frequency was and the mode shape was obtained, below is schematic diagram of the stator. 

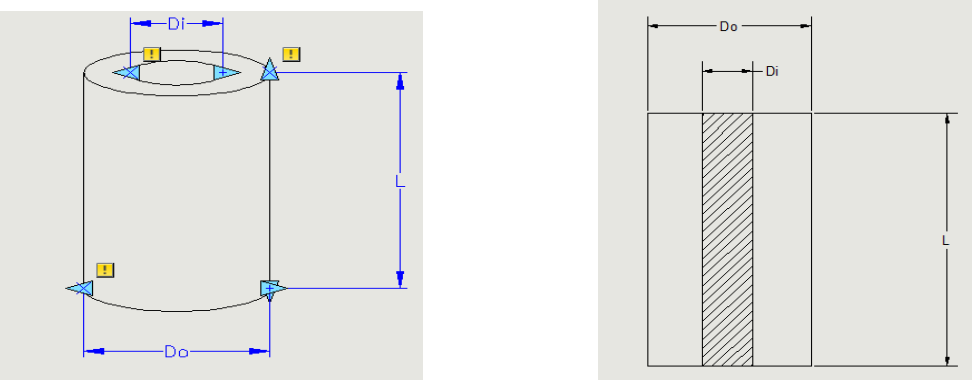

Fig. 1. The schematic diagram of the bar-type stator

\subsection{Formation Of Traveling Wave In The Stator}

The stator of Double Rotor Bar-Type Traveling Wave Rotary Ultrasonic Motor (DBTRUM) is a hollow bar with inner diameter d, outer diameter D and length 1. from Euler-Bernoulli theory, the equation governing the natural bending vibration of this bar is as follows.

$$
\begin{aligned}
& p_{i}= \frac{\lambda_{i}^{2}}{l^{2}} a=\frac{\lambda_{i}^{2}}{l^{2}} \sqrt{\frac{E I}{\rho_{v} A}}, \\
& f_{i}=\frac{\lambda_{i}^{2}}{2 \pi l^{2}} \sqrt{\frac{E I}{\rho A}}=\frac{\lambda_{i}^{2}}{2 \pi l^{2}} \sqrt{\frac{E\left(D^{2}+d^{2}\right)}{16 \rho_{v}}}
\end{aligned}
$$

Where,

$p_{i}$ : The natural frequency of vibration, $\rho_{v}$ : The density of material of the bar, A: cross-sectional area of the bar, I: Second moment of area, E: modulus of elasticity of the material,

$\lambda_{i}$ : Characteristic root, D: Outer diameter, d: Inner diameter

In order to get a "pure" traveling wave in the stator the following conditions must be attained, two orthogonal "pure" modes (standing waves) with the same frequency and shape must be induced on the stator.

If any of any of the conditions is not satisfied, there will be no "pure" traveling wave in the stator, therefore when the exciting signals with $\pi / 2$ phase shift in time are imposed on the two sets of specifically polarized piezoelectric ceramic pieces respectively, then two phase modal responses on the stator can be obtained with $\pi / 2$ phase shift both in space and time. Below is the piezoelectric ceramics arrangements and the polarization method on the stator that produces the traveling wave.
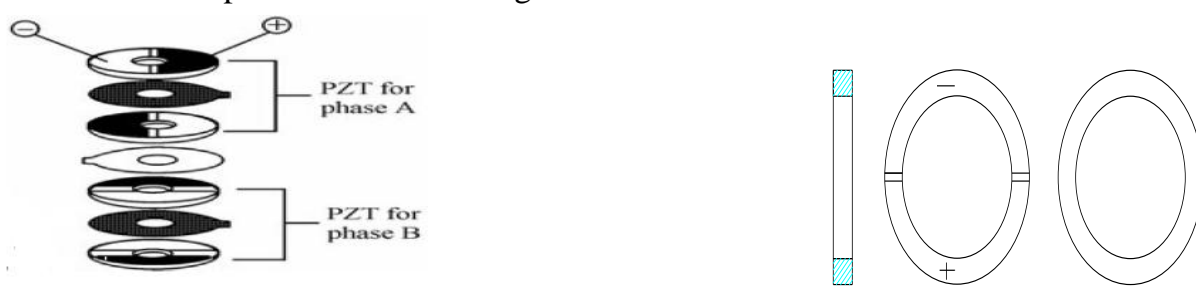

Fig. 2b PZT poling arrangement

\section{System Design}

The design of stator of DBTRUM (SDOF) will be carried out, both theoretically (using the application of mathematical formulae of the bending vibration mode) and also simulated using finite element method software (using ANSYS software), the required mode shape and the vibrating frequency will be obtained. The stator in figure 1 will be used throughout this analysis, the operating frequency of a traveling wave rotary ultrasonic motor (TRUM) lies in the range from $30 \mathrm{kHz}$ to $50 \mathrm{kHz}$, for TRUM with smaller radius than $40 \mathrm{~mm}$.

\subsection{Theoretical Design of the Bar-type Stator}

The bar-type stator of the DBTRUM will be designed based upon theoretical analysis of the first bending vibration mode, the theoretical frequency (calculated frequency) will be obtained without the considering the application of piezoelectric ceramic using the bending vibration equation, in this analysis, the stator will vibrate freely without any support or constraint. 
Consider the stator in figure 1 above where $\mathrm{D}$ is the outer diameter of the stator which is the main diameter of the bar, $\mathrm{d}$ is the diameter of the hollow part and 1 is the stator's length. For the bar in figure 1, the theoretical frequency of vibration of the first bending mode will be calculated from the following equation.

$$
f_{i}=\frac{\lambda_{i}^{2}}{2 \pi l^{2}} \sqrt{\frac{E I}{\rho A}}
$$

For a circular hollow cross-sectional area, the second moment of area I and the cross-sectional area A can be obtained from the following equations.

$$
I=\frac{\pi\left(D^{4}-d^{4}\right)}{64}, A=\frac{\left(D^{2}-d^{2}\right) \pi}{4}
$$

Where, $\mathrm{D}=$ outer diameter and $\mathrm{d}=$ inner diameter, There frequency of the bending vibration is as follows.

$$
f_{i}=\frac{\lambda_{i}^{2}}{2 \pi d^{2}} \sqrt{\frac{E\left(D^{2}+d^{2}\right)}{16 \rho_{v}}} \quad \text { For first free bending mode, the characteristic root } \lambda_{i}=4.730 ;
$$

Consider the bar in fig. 1 having the following dimensions,

$l=0.039 \mathrm{~m}, \mathrm{D}=0.030 \mathrm{~m}, \mathrm{~d}=0.011 \mathrm{~m}$,

The first frequency of bending vibration is calculated below.

$$
f_{i}=\frac{(4.730)^{2}}{2 \pi(0.039)^{2}} \sqrt{\frac{2 \times 10^{11}\left(0.03^{2}+0.011^{2}\right)}{16 \times 7800}}=94696.5 \mathrm{~Hz}
$$

\section{Finite Element Simulation Of The Stator Of Dbtrum}

3.1 Design of Stator of the Double Rotor Bar-type Traveling Wave Ultrasonic Motor with Piezoelectric Ceramic (PZT)

Analytical solutions to an elastic body vibration equation are limited to only simple geometries with specific boundary conditions. In most other cases, numerical methods are used instead to obtain approximate solutions. The finite element method (FEM) is the most effective one, of which some highly sophisticated software such as ANSYS, NASTRAN, ATILA, etc. is based on. Therefore, all the simulation and analysis in this thesis will be carried out using ANSYS software.

The stator of the DBTRUM will be simulated without taking the piezoelectric ceramic actuator into consideration, the frequency of the first bending vibration mode obtained from the simulation will be analyze and compared with the result obtained from the theoretically calculated result. The simulation will be carried out using ANSYS software. Below are the mode shape, maximum displacement and frequency obtained from the simulation.

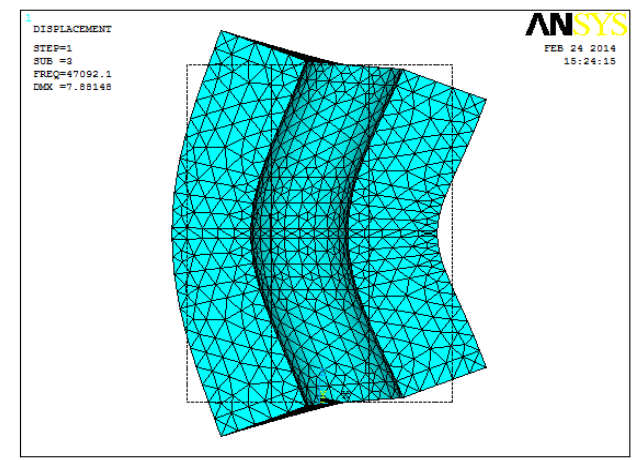

Fig. 3 First bending vibration mode of the stator of DBTRUM (without PZT attached)

\subsection{Design of Stator of the Double Rotor Bar-type Traveling Wave Ultrasonic Motor with Piezoelectric Ceramic (PZT) Attached}

The use of piezoelectric ceramic element to generate vibration as a result of inverse piezoelectric effect, which excites the stator to produce forced vibration response make the results obtained from this simulation more proximate to that of the real system. 
Modal analysis of a stator of Double Rotor Traveling Wave Rotary Ultrasonic Motor....

The piezoelectric ceramic is fixed at the middle of the stator so that it induces vibration on the stator. The bending vibration mode was obtained, and also the frequency of the bending vibration mode was obtained using the ANSYS software as follows.

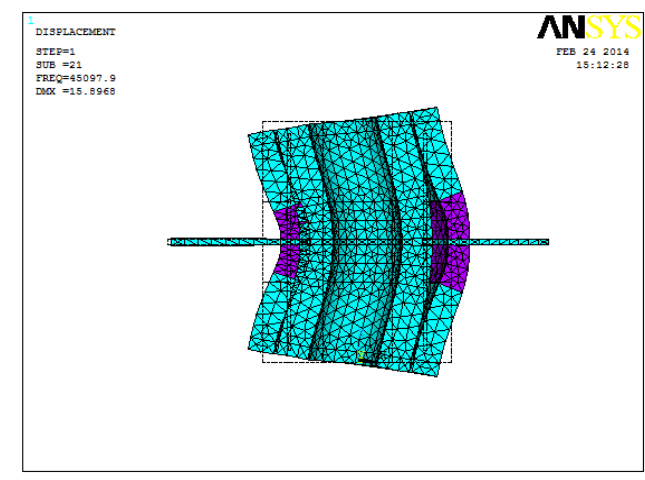

Fig. 4 First bending vibration mode of the stator of DBTRUM (with PZT attached)

\section{Results}

Below are results obtained from the theoretical (mathematical), finite element simulation (without PZT) and finite element simulation (with PZT)

TABLE I

\begin{tabular}{|c|c|c|}
\hline ANASYS TYPE & MODE SHAPE & MODAL FREQUENCY f(Hz) \\
\hline $\begin{array}{c}\text { Theoretical (mathematical) } \\
\text { modal analysis }\end{array}$ & First bending mode & 94696 \\
\hline $\begin{array}{c}\text { Finite element simulation } \\
\text { (without piezoelectric } \\
\text { ceramic). } \\
\text { (using ANSYS software) }\end{array}$ & First bending mode & 47092 \\
\hline $\begin{array}{c}\text { Finite element simulation } \\
\text { (with piezoelectric ceramic). } \\
\text { (using ANSYS software) }\end{array}$ & First bending mode & 45098 \\
\hline
\end{tabular}

\section{Discussion}

As analytical solutions to an elastic body vibration equation are limited to only simple geometries with specific boundary conditions. Numerical methods are used to obtained approximate solutions. From the results obtained in this analysis, it can observed that the frequency calculated from the theoretical modal analysis is $94696 \mathrm{~Hz}$, this is too higher, as it is out of the required range for this system (30kHz to $50 \mathrm{kHz})$, this is as a result of approximations made in the initial and boundary conditions, also the effect of piezoelectric ceramic was not considered. This analysis is used to obtain an approximate result.

The finite element method (FEM) is the most effective method of obtaining the solution to an elastic body vibration. The first bending vibration modal frequency obtained from the finite element simulation without taking the effect of piezoelectric ceramic into consideration is $47092 \mathrm{~Hz}$; this result is within the required frequency range for the design of this system which is $(30 \mathrm{kHz}$ to $50 \mathrm{kHz})$. By comparing the finite element simulation result with that of the theoretically calculated one, it can be observed that the finite element simulation result is more accurate because of its proximity to the required designed frequency, only that piezoelectric ceramic was neglected which is an important part of any ultrasonic motor, as it is required to generate forced vibration in the stator by the inverse piezoelectric effect. Therefore, this simulation is used to obtain an approximate solution.

The application of piezoelectric ceramic in the finite element simulation to generate vibration in stator provides much more accurate results in modal analysis. The first bending vibration mode obtained in this simulation as the effect piezoelectric ceramic is considered is 45098; this is within the designed frequency range $(30 \mathrm{kHz}$ to $50 \mathrm{kHz})$ of traveling wave rotary ultrasonic motors with radius smaller than $40 \mathrm{~mm}$.

\section{Conclusion}

This paper present the modal analysis of the stator of DBTRUM both theoretically and FEM using ANSYS software, considering the result obtained from the finite element simulation with piezoelectric ceramic effect 
Modal analysis of a stator of Double Rotor Traveling Wave Rotary Ultrasonic Motor....

included, the frequency obtained for the first bending vibration mode is $45098 \mathrm{~Hz}$, this is within the required frequency ranges for traveling wave motors, therefore, the prototype system will be designed and produced on the basis of this finite element simulation, only that some few adjustments will be made on the prototype. It can be concluded that, DBTRUM can be implemented as the simulation result is within the designed range $(30 \mathrm{kHz}$ to $50 \mathrm{kHz}$ ) for a TRUM stator with radius less than $40 \mathrm{~mm}$.

\section{REFERENCES}

[1] Chunsheng Zhao, Ultrasonic Motors (Technologies and Application), Beijing: science press. 2011

[2] Dongzhe Bai,et al. An Ultrasonic Motor Driven by the Phase-Velocity Difference Between Two Traveling Waves, IEEE transactions on ultrasonic, ferroelectrics, and frequency control, vol. 51, no. 6, June 2004

[3] Kenji Ucheno, Piezoelectric Ultrasonic Motors, A journal of Ultrasonic motors using piezoelectric resonant vibration, 7 (1998), 273-285.

[4] Kenjiro Takemura, Nobuyuki Kojima, Takashi Maeno. Development of Bar-Shaped Ultrasonic Motor for Multi - Degree of Freedom Motion, A journal of Bar-type Ultrasonic Motors for the application in dexterous robot arms and manipulators. 2011

[5] Nishant Venkatesan. An evaluation of the traveling waves ultrasonic motor for force feedback applications, University of Kentucky Master's degree Theses, February 13, 2009.

[6] Weishan Chen, Junkao Liu, Shengjun Shi, study and test of a double-rotor ultrasonic motor with symmetrical longitudinal-torsional converter. 18th IEEE International Symposium on Applications of Ferroelectrics, ISAF 2009.

[7] University of Nanjing Aeronautics. Double-rotor bending mode linear ultrasonic motor. Patent Family Members (1; CN), CN 201656809 U, (24-Nov2010).

[8] Harbin Engineering University. Large-moment double-rotor stress type longitudinal-torsional composite ultrasonic motor and electric excitation method thereof. Chinese patent, CN 101719735 A. Feb 1, 2012. (translated from Chinese)

[9] Markus G. Bauer, Design of a Linear High Precision Ultrasonic Piezoelectric Motor, North Carolina State University Doctorate's dissertation, 2001 .

[10] Burhanettin Koc, Dilek Başaran, Tayfun Akin, Abdulkadir Erden, Design of a Piezoelectric Ultrasonic Motor for Micro-Robotic Application, English Journal of Piezoelectric Ultrasonic Motor, Tubitak through Contract No: MISAG-209, Turkey. (2007)

[11] Chee Kian Lim Siyuan He I-Ming Chen Song Huat Yeo. A Piezo-on-Slider Type Linear Ultrasonic Motor for the Application of Positioning Stages, English Journal Of Piezoelectric Ultrasonic Motor, School of Mechanical and Production Engineering, Nanyang Technological University, Singapore. (1999)

[12] Serra Cagatay, Kenji Uchino and Burhanettin Koc. Ultrasonic Motor, English Journal Of Piezoelectric Ultrasonic Motor, International Center for Actuators and Transducers, Materials Research Institute, the Pennsylvania State University, University Park, PA, 16802. Atilim University, Mechatronics Engineering, Ankara, Turkey. (1998)

[13] Vom Fachbereich Mechanik. Dynamics of Ultrasonic Motors, Darmstadt University of Technology, Doctorate's dissertation (2003).

[14] Vytautas Ostaseviciusa, Arvydas Palevicius, et al. Hybrid numerical - experimental optical investigation of the contact zone of ultrasonic motors, a journal of USM, International Studies Center of Kaunas University of Technology, Mickeviciaus 37, Kaunas, Lithuania, LT-44244. (2005).

[15] Tomonobu Senjyu, Tomohiro Yoshida, Katsumi Uezato, and S. K. Panda. Position Control of Ultrasonic Motors Using Dead-Zone Compensation with Fuzzy Neural Network, a journal of USM, Faculty of Engineering, University of the Ryukyus Senbaru Nishihara-cho Nakagami Okinawa 903-0213(2003), JAPAN.

[16] Fan Zhang, Weishan Chen, Junkao Liu, and Zhisong Wang. Bidirectional Linear Ultrasonic Motor Using Longitudinal Vibrating Transducers, a journal of linear USM, IEEE transactions on ultrasonic, ferroelectrics, and frequency control, vol. 52, no. 1, January 2005.

[17] Hamed Mojallali, et al. Free Stator Modeling of a Traveling Wave Ultrasonic Motor, a Proceedings of the 13th Mediterranean Conference on Control and Automation Limassol, Cyprus, June 27-29, 2005.

[18] Frederic Giraud, Betty Lemaire-Semail, et al. Precise Position Control of a Travelling Wave Ultrasonic Motor, Laboratory of electrotechnics and power electronics of Lille Avenue Paul Langevin. (2005)

[19] Masahiko Tominaga, et al. An Ultrasonic Linear Motor Using Ridge-Mode Traveling Waves, IEEE transactions on ultrasonics, ferroelectrics, and frequency control, vol. 52, no. 10, October 2005.

[20] Oleksiy Vyshnevskyy, Sergej Kovalev, and Wladimir Wischnewskiy. A Novel, Single-Mode Piezoceramic Plate Actuator for Ultrasonic Linear Motors, IEEE transactions on ultrasonics, ferroelectrics, and frequency control, vol. 52, no. 11, November 2005.

[21] Izhak Bucher. Estimating the ratio between travelling and standing vibration waves under non-stationary conditions, Journal of Sound and Vibration 270 (2004) 341-359

[22] A. Frangi et al. Finite element modelling of a rotating piezoelectric ultrasonic motor, Ultrasonics 43 (2005) $747-755$.

[23] Jong-Sung Park, Sang-Tae Kim, et al. Ultrasonic Linear Motor using L1-B4 Mode and its Analysis, Japanese Journal of Applied Physics, Vol. 44, No. 1A, 2005, pp. 412-416 